
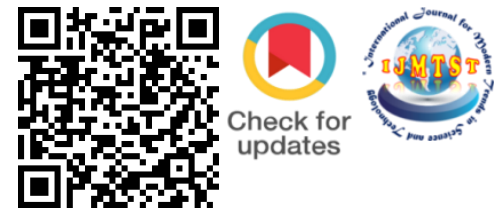

\title{
Social Distancing Detection Using Open CV and Yolo Object Detector
}

\author{
Akanksha Shukla ${ }^{1} \mid$ Ishani Garkoti ${ }^{2} \mid$ Amisha Mittal $^{3} \mid$ Binit Choudhary ${ }^{4} \mid$ Dr. Preety Verma Dhaka \\ 1,2,3,4,5 Department of IT, Dr. Akhilesh Das Gupta Institute of Technology \& Management, Delhi, India
}

\section{To Cite this Article}

Akanksha Shukla, IshaniGarkoti, Amisha Mittal, Binit Choudhary and Dr. Preety Verma Dhaka,"Social Distancing Detection Using Open CV and Yolo Object Detector", International Journal for Modern Trends in Science and Technology, Vol. 07, Issue 01, January 2021, pp.- 93-95.

\section{Article Info}

Received on 22-November-2020, Revised on 18-December-2020, Accepted on 28-December-2020, Published on 07 -January-2021.

\section{ABSTRACT}

Lately, social distancing has become a trending term, more because of the COVID-19 pandemic that has affected the entire world causing more than 1 million deaths. The world we lived in a few months prior is completely different from what it is now. The lack of any antidotes and the absence of immunity, capable of fighting off the virus has made humans more undefended. Hence, Social Distancing is the only best option for us to protect ourselves from diseases, not limited to COVID-19, that may be transmitted through human contact. Social distancing is a technique that may be used to reduce the rate of new cases during a pandemic outbreak. This publication is focusing on surveillance of public places and detecting whether the people are maintaining social distancing or not. It explains the development of technology through the use of AI-based procedures to detect whether the social distancing norm is followed or not, in any public video stream.

The software embedded can distinguish between a person maintaining social distance (marked green) and a person who is not (marked red) and will also keep a count of incidents where social distancing was not followed.

KEYWORDS: Social Distancing, Covid-19, Computer Vision, YOLO Object detection, Python, OpenCV, Deep Learning, Social health, Social surveillance, Coronavirus

\section{INTRODUCTION}

Social distancing is a method used to control the spread of contagious diseases.

As the name suggests, social distancing implies that people should physically distance themselves from one another, reducing close contact, and thereby reducing the spread of a contagious disease (such as coronavirus). Social distancing is arguably the most effective non pharmaceutical way to prevent the spread of a disease - by definition, if people are not close together, they cannot spread germs.
Using technology, more specifically the AI -based, we have developed a mechanism, which can be implemented on a video stream of any locality. It may distinguish people who are following the social distancing, and those who are not. 


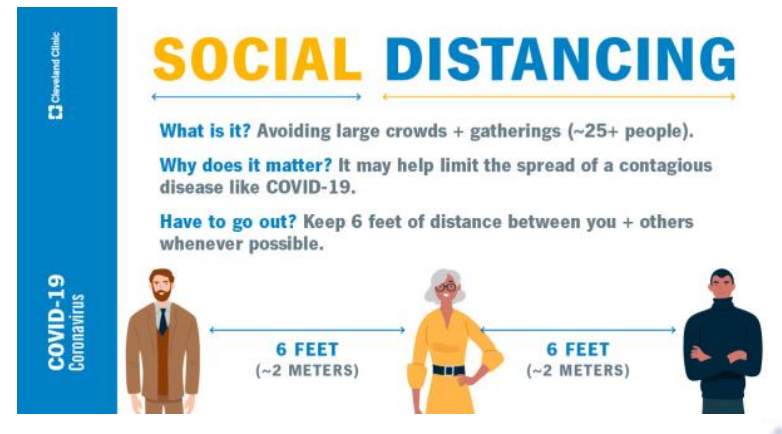

\section{A. Deep Learning}

Deep Learning is a subfield of machine learning. It was inspired by the structure and function of the brain called artificial neural networks and concerned with similar algorithms.

These algorithms inspired by the human brain, learn from large amounts of data just like we, humans, learn from experience.

The Deep Learning algorithms repeat the process each time, tweaking it a little to improve the outcome.

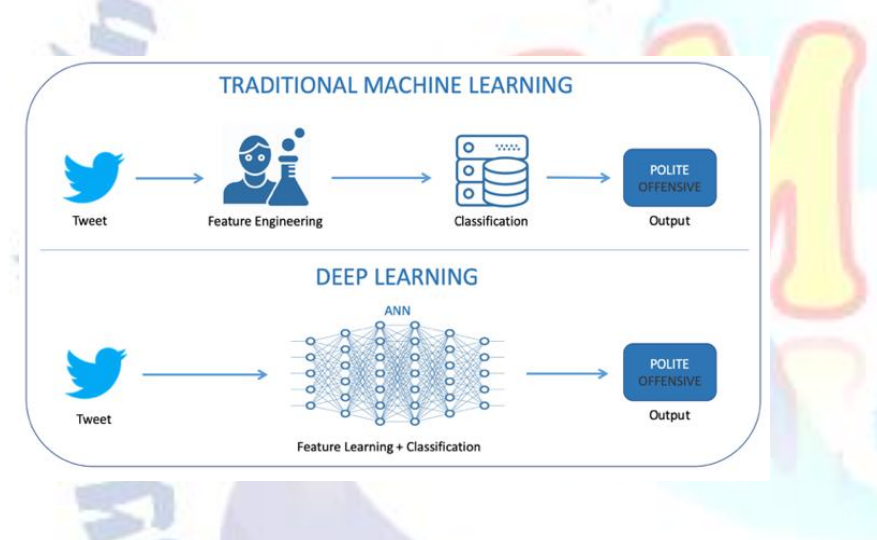

\section{B. Computer Vision}

Computer Vision, also abbreviated as CV, is a field of study that aims to develop techniques that help computers "see" and understand the content of digital images. These digital images can be in the form of photographs and videos. The importance of computer vision is basically in the problems that it can solve. Computer Vision is a technology that makes the interaction between the digital world and the physical world possible. It is also used in applications involving facial recognition. Facial Recognition implies a technology that enables computers to link people's face images to their identities. Computer vision algorithms does this by detecting facial features in given images and then compares them with databases of face profiles.

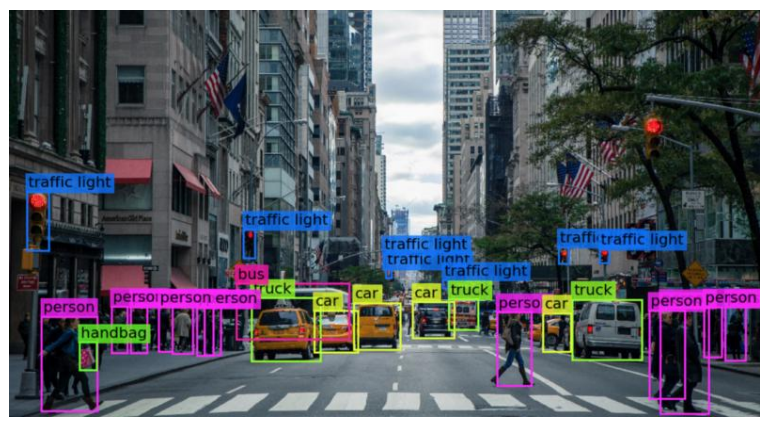

\section{OpenCV}

OpenCV, also called 'Open-Source Computer Vision Library' is, as the name suggests, an open-source library for computer vision, machine learning, and image processing. It plays a major role in the real-time operation, important in today's systems. One can process images and videos to identify objects, faces, or even the handwriting of a human using the OpenCV. The data can be in several forms; it can be in the form of video sequences, views from multiple cameras, multidimensional data from a 3D scanner, or even medical scanning device.

\section{Yolo Object Detection}

YOLO, a short term for 'You Only Look Once', is a real-time object detection algorithm. It is one of the most effective object detection algorithms and has been involved in most innovative ideas that have and are coming from the research community associated with computer vision. Object detection is a critical capability of autonomous vehicle technology and is an area of computer vision that has become more efficient than before. YOLO is a clever convolutional neural network $(\mathrm{CNN})$ which detects objects in real time.

The algorithm applies a single neural network to a full image, and then divides the image into regions. Further, it predicts the bounding boxes and probabilities for each region. Lastly, these bounding boxes are weighted by the predicted probabilities.

\section{BACKGROUND SURVEY AND RELATED WORK}

\section{A. Motivation}

The social distancing is playing an important role during the ongoing COVID-19 pandemic. The first case of a COVID-19 affected patient was reported in Wuhan, China. Within one month of the outbreak, the number of positive cases and deceased rose at an exponential rate. Later, with the implementation of social distancing, the count 
reduced drastically, and was later adopted worldwide.

\section{FUtURE SCOPE}

This publishing is focusing on surveillance of public places and detecting whether the people are maintaining social distancing or not. Social Distancing is the only best option for us to protect ourselves from diseases, not limited to COVID-19, where no medicinal antidote has been prepared, and that may be transmitted through human contact. The paper explains the development of a technology through use of AI based procedures to detect whether the social distancing norm is followed or not, in any public video stream.

The software embedded can distinguish between a person maintaining social distance (marked green) and a person who is not (marked red). We will also keep a count of incidents where social distancing was not followed.

\section{A. Objective}

To develop a technology that may trace whether the social distancing rules are obeyed or not.

To control any outspread of any future contagious disease.

To develop a Privacy-friendly software; no PII is used or stored.

To Preserve the work continuity while also keeping the people safe.

To Increase confidence in people moving in public places.

\section{B. Future scope of the system}

With advanced technical updates, the system may be capable to trace and detect the violations from an aerial view.

\section{Conclusion}

Based on the results obtained, we can see that social distancing detector is correctly marking people who violate social distancing rules. While all the people who are following the norm are enclosed in green rectangular boxes, people violating the social distancing norm are enclosed in a red rectangular box. Nowadays, social distancing along with other basic sanitary measures are very important to keep

the spread of the pandemic (Covid-19) as slow as possible. Along with the color representation, the count, that is the number of the times the norm is violated is also displayed based on the video stream.
The algorithm is used to analyze social distancing in a public area and carry out necessary actions to better deal with the pandemic. The project has been completed using the implementation of computer language technology onto a video stream to develop an application working as a social distancing detector, also capable of keeping count of norm violation. This also included

the testing of the application, cross-checking of the data until a satisfactory, required, correct and good result was obtained.

\section{REFERENCES}

[1] Rucha Visal, Atharva Theurkar, Bhairavi Shukla," Monitoring Social Distancing for Covid-19 Using OpenCV and Deep Learning" 2020 International Research Journal of Engineering and Technology (IRJET).

[2] Vinitha.V,Velantina.V,"Social distancing detection system with artificial intelligence using computer vision and deep learning" 2020 International Research Journal of Engineering and Technology (IRJET).

[3] Yew Cheong Hou, MohdZafriBaharuddin, Salman Yussof, SumayyahDzulkifly," Social Distancing Detection with Deep Learning Model" 2020 8th International Conference on Information Technology and Multimedia (ICIMU)

[4] Narinder Singh Punn, Sanjay Kumar Sonbhadra and Sonali Agarwal," Monitoring COVID-19 social distancing with person detection and tracking via fine-tuned YOLO v3 and Deepsort techniques" 